\title{
Self-starvation and activity stress in Brattleboro rats
}

\author{
HELEN M. MURPHY and CYRILLA H. WIDEMAN \\ John Carroll University, Cleveland, Ohio
}

\begin{abstract}
In order to examine the susceptibility of Brattleboro rats to self-starvation and activity stress, as evidenced by ulcer formation, four conditions were studied: (1) ad-lib access to food and water in an individual home cage, (2) ad-lib access to food and water in an activity-wheel cage, (3) ad-lib access to water and 1-h access to food in an individual home cage, and (4) ad-lib access to water and 1-h access to food each day in an activity-wheel cage. In the first two conditions, neither Brattleboro nor control animals developed ulcers. In the third condition, some rats in each group developed pits in the glandular portion of the stomach. Significant differences appeared in the fourth condition. Brattleboro rats developed more stomach pathology in both the nonglandular and glandular portions of the stomach than controls. The results were not due to variations in running behavior. The data indicate that the lack of vasopressin or physiological results of this deficit render Brattleboro rats more sensitive to self-starvation and activity stress than control animals.
\end{abstract}

Brattleboro rats (DI) which lack the hormone vasopressin are genetic mutants of the Long-Evans (LE) strain. The DI rat has the pathological disorder known as diabetes insipidus (Valtin \& Schroeder, 1964). Our laboratory has reported that DI animals are more susceptible to stress ulcer formation under conditions of restraint and restraint plus intermittent shock than LE animals (Wideman \& Murphy, 1983). The etiology of ulcer formation may vary. In addition to the restraint stress ulcer, activity stress ulcers (Paré, 1974, 1975, 1976, 1977a, 1977b; Paré \& Houser, 1973; Paré, Natelson, Vincent, \& Isom, 1980) and starvation ulcers (Paré \& Temple, 1973; Pfeiffer, Debro, \& Muller, 1966; Robert \& Nezamis, $1958 \mathrm{a}, 1958 \mathrm{~b})$ have also been demonstrated in normal animals.

Routtenberg and Kuznesof (1967) examined animals on 23-h food-deprivation cycles and housed in activity wheels. They noted that these animals were unable to maintain body weight and died. This phenomenon was termed "self-starvation." Their working hypothesis was that deprivation leads to hunger and weight loss, which cause overactivity in certain lateral and posterior hypothalamic nuclear systems. One consequence of this overactivity is an increase in locomotor activity. They further hypothesized that hypothalamic activity and locomotor activity generate certain products which act as signals to the hypothalamus that are interpreted as food and that, therefore, depress food intake. Using a similar paradigm, Paré and Houser (1973) showed that if rats were housed in activity-wheel cages, allowed contin-

The author's mailing address is: Department of Psychology, John Carroll University, 20700 North Park Boulevard, Cleveland, Ohio 44118. uous access to the running wheel, and fed only $1 \mathrm{~h}$ each day, some of the animals would die within 4 to 12 days and reveal extensive lesions in the glandular stomach. They termed these lesions "activity-stress ulcers." Pfeiffer, Debro, and Muller (1966) have reported that starvation of older rats results in the development of ulcers in the nonglandular portion of the stomach. These ulcers have been designated as "starvation ulcers." The purpose of the present study was to determine the susceptibility of DI animals to self-starvation and activity stress utilizing the criterion of ulcer induction.

\section{METHOD}

\section{Animals}

The subjects utilized were $\mathbf{4 0}$ male DI and $\mathbf{4 0}$ male LE rats. It has been suggested by Valtin (1982) that the best control for a homozygous DI rat is a LE rat from the same colony that is used periodically for outbreeding and the production of "new" heterozygotes. This use of the LE rat avoids excessive inbreeding and provides controls that are genetically similar to the animals that carry the Brattleboro gene. It has also been proposed by Murphy and Wideman (1982) that a control group of LE rats be employed in experiments utilizing Brattleboro rats. In the present study, the rats were 18 weeks old when beginning the experiment. All animals were placed in alternating periods of light and darkness each day, with the lights going on at 8:00 a.m. and off at 8:00 p.m. Room temperature was maintained at $72^{\circ}-74^{\circ} \mathrm{F}$. With the knowledge that LE animals weighed significantly more than DI animals of the same age, the two groups were equated as closely as possible for body weight before the subjects were assigned to one of four treatment groups: (1) ad-lib access to food and water in an individual home cage, (2) ad-lib access to food and water in an activity wheel cage, (3) ad-lib access to water and 1-h access to food each day in an individual home cage, and (4) ad-lib access to water and $1-h$ access to food each day in an activity wheel cage.

\section{Procedure}

Ten DI and 10 LE rats were utilized in each of the treatment groups. Rats in Groups 2 and 4 were individually housed in 
running-wheel activity cages (Wahmann Manufacturing Co., Baltimore). Each activity wheel had an adjoining cage, which measured $25 \times 15 \times 13 \mathrm{~cm}$, and a sliding door, which could block access to the activity wheel. Animals in the fourth group were habituated to the activity-wheel cage for 7 days before the testing began. During the habituation period, the rats had ad-lib access to food and water in the activity-wheel cage and the sliding door was always open. The testing period (1-h access to food each day) was 9 days. Access to the running wheel was blocked during the feeding period. During this time, body weight, water intake, and number of revolutions were recorded each day. At the end of the testing period, subjects from all four groups were sacrificed by decapitation and the stomachs were removed and inspected for ulcers.

Nonglandular (rumenal or forestomach) lesions were characterized first by a tremendous edema of the forestomach wall; later, necrotizing ulcers developed and the reaction became more severe. Glandular ulcers were defined as acute gastric lesions that were reddish brown in color. Some lesions were irregular and linear and tended to parallel folds of the glandular mucosa, whereas other lesions were circumscribed and focal.

\section{Results}

In the conditions in which animals were allowed ad-lib access to food and water in the home cage (Condition 1) and ad-lib access to food and water in the activity-wheel cage (Condition 2), no animals developed ulcers. In the condition in which animals were restricted to $1 \mathrm{~h}$ of feeding each day in the home cage (Condition 3), five DI and two LE animals developed small red pits in the glandular portion of the stomach. These pits were not large enough to be measured and classified as true ulcers. In the condition in which animals were restricted to $1 \mathrm{~h}$ of feeding each day in the activity-wheel cage (Condition 4), all DI and LE animals developed ulcers. All animals developed lesions in the nonglandular portion of the stomach. The evaluation of forestomach lesions was based upon the method utilized by Robert and Nezamis (1958b). This method examines the forestomach wall and considers edema as well as true ulcers. Table 1 summarizes the evaluation of the nonglandular stomach of all animals in the fourth condition. Utilizing a $t$ test, it was determined that DI animals had significantly more edema than LE animals $(p<.001)$. DI animals also had significantly more ulcers than LE animals in the nonglandular stomach $(p<.001)$.

The glandular portion of the stomach showed more pathology in DI than in LE animals $(p<.01)$. All $10 \mathrm{DI}$ and only $5 \mathrm{LE}$ animals showed ulceration in this part of the stomach. Table 2 shows the mean length of ulcers and the mean number of ulcers per rat in the glandular region of the stomach.

With 1-h access to food in the home cage (Condition 3), DI animals had a body-weight loss of $20.1 \%$ and LE animals had a body-weight loss of $13.1 \%$. DI animals lost significantly more body weight than controls $(p<.01)$. In Condition 4, DI animals also lost significantly more body weight than LE animals $(23.6 \%$ vs. $18.2 \%, p<.01)$ in a comparison between
Table 1

Stomach Pathology in the Nonglandular Portion of the Stomach

\begin{tabular}{lrc}
\hline \multicolumn{1}{c}{ Stomach Pathology } & DI & LE \\
\hline Edema (\% of Animals Affected) & 100 & 100 \\
Edema (Mean \% of Area Affected) & 88 & $37 *$ \\
Ulcers (\% of Animals Affected) & 100 & 100 \\
Ulcers (Mean Number/Rat) & 10.3 & $2.7^{*}$ \\
\hline Note-DI = Brattleboro rats; LE = Long-Evans rats. & ${ }^{*} p<.001$
\end{tabular}

Table 2

Ulcers in the Glandular Portion of the Stomach

\begin{tabular}{ccc}
\hline Ulcers & DI & LE \\
\hline \% of Animals Affected & 100 & 50 \\
Mean Length (mm) & 2.0 & $0.3^{*}$ \\
Mean Number/Rat & 5.3 & $1.0^{*}$ \\
\hline Note-DI = Brattleboro rats; $L E=$ Long-Evans rats. & ${ }^{*} p<.01$
\end{tabular}

the first day of habituation and the last day of testing. Figure 1 shows the mean body weight of the animals in Condition 4 during the 7 days of habituation and 9 days of testing. As noted previously in several studies, LE animals that are the same age as DI animals weigh significantly more than the DI animals.

In Condition 3, DI animals drank $18.8 \%$ less water on the last day of the testing period than on the first day of the habituation period and LE animals drank

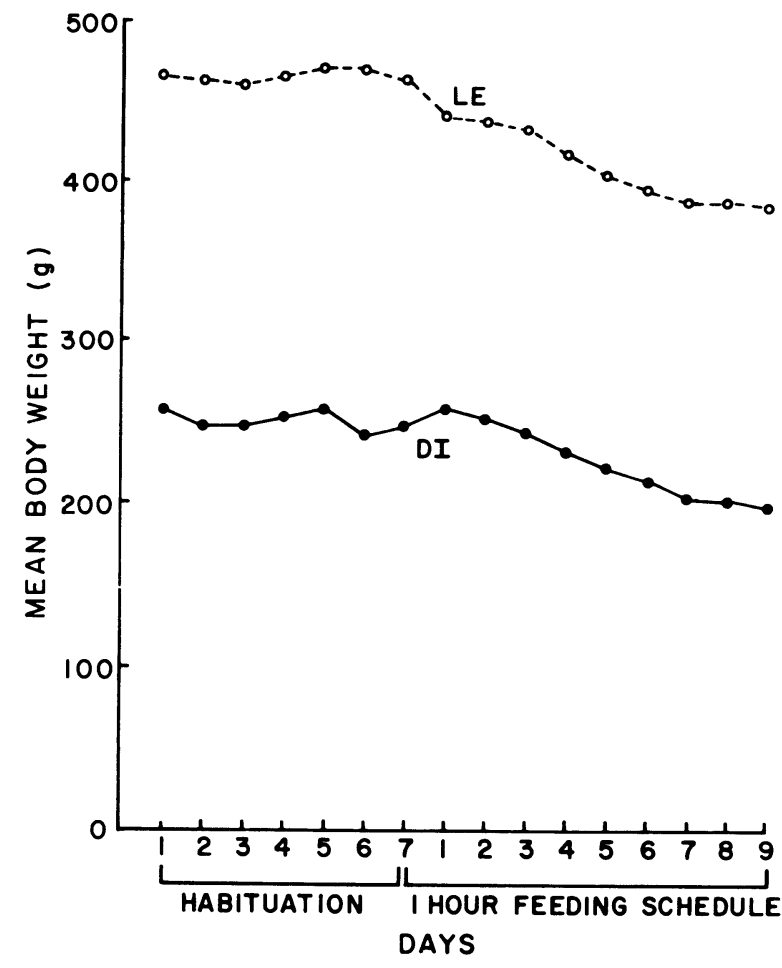

Figure 1. Mean body weight in DI and LE animals during the habituation and test periods. 
$15.8 \%$ less water on the last day of the testing period than on the first day of habituation. There were no significant differences between the two groups in the percentage drop of water intake. In condition 4 , all animals drank significantly less water during the testing sessions than during the habituation period. DI animals drank $54.0 \%$ less on the last day of testing than on the first day of habituation, and LE animals drank $33.0 \%$ less water on the last day of testing than on the first day of habituation. Figure 2 shows the mean intake of water of these animals during the habituation and testing periods. For both groups, the decrease of water intake that occurred between the habituation and testing periods was significant at the .001 level. Although both groups decreased their water intake during the testing period, DI animals still drank significantly more water than LE animals $(p<.02)$. Note the dramatic drop in DI-animal water intake during the testing sessions.

In Condition 4, there were no significant differences in running behavior between the two groups in either the habituation or the testing sessions. Both groups ran significantly more in the testing session than in the habituation period $(p<.001)$. Figure 3 shows the running behavior of the two groups during both the habituation and testing sessions.

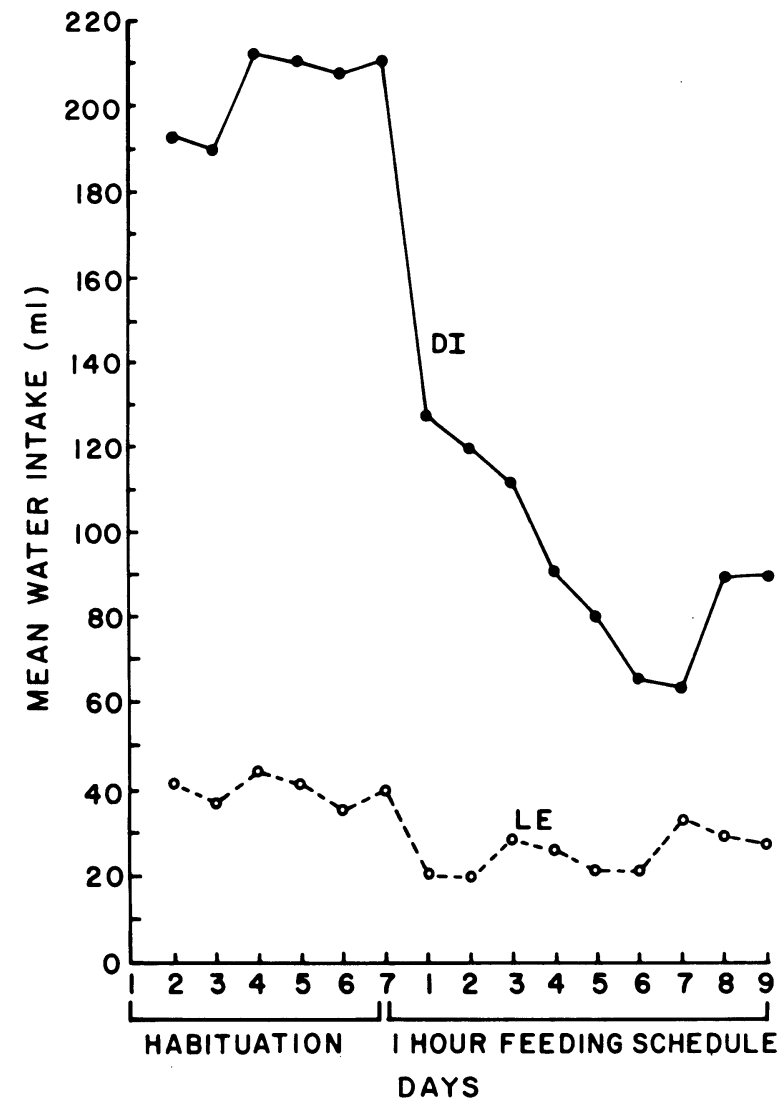

Figure 2. Mean water intake in DI and LE animals during the habituation and test periods.

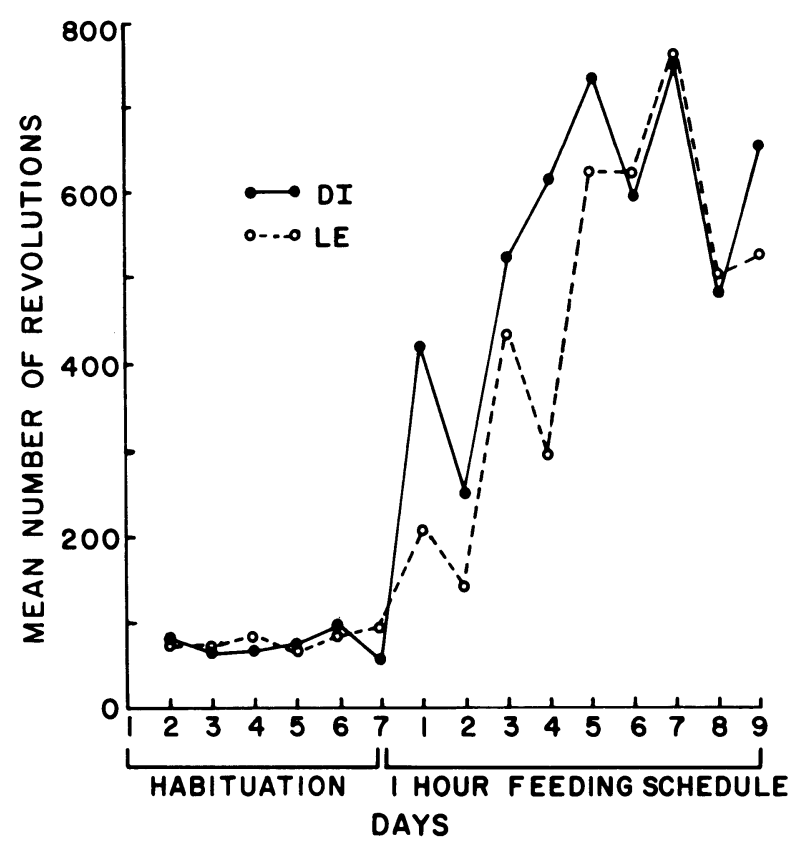

Figure 3. Mean number of revolutions of the activity wheel for DI and LE animals during the habituation and test periods.

\section{DISCUSSION}

The results of this experiment show that when DI and LE rats are given a limited time to feed and 23-h/day access to a running wheel, they increase their running behavior, lose body weight, and develop ulcers. In contrast, DI and LE animals given ad-lib access to food and water in an individual home cage or in an activity-wheel cage do not lose body weight and do not develop ulcers. Both DI and LE animals that are given ad-lib access to water and 1-h access to food in the home cage lose body weight and develop small pits in the glandular portion of the stomach. In the condition of ad-lib access to water and 1-h access to food in the activity-wheel cage, DI animals developed ulcers in both the nonglandular and glandular portions of the stomach. Under the same condition, LE animals developed ulcers in the nonglandular portion of the stomach in all cases and developed ulcers in the glandular portion of the stomach in only half of the cases. DI animals showed significantly more ulceration than LE controls in both the nonglandular and glandular portions of the stomach.

In previous research, Wideman and Murphy (1983) demonstrated that under conditions of restraint and restraint plus intermittent shock, DI animals, as compared with controls, showed an exaggerated response to the stressor. Glandular ulcers were evident in the stomachs of both DI and LE groups, with significantly more lesions observed in the DI animals. The present experiment demonstrates that DI animals develop more stomach pathology than LE ani- 
mals, since DI animals develop more ulcers than controls in both the nonglandular and glandular portions of the stomach. Nonglandular ulcers were lacking in the restraint and restraint-plus-intermittent shock experiment. The extent of nonglandular ulceration was unexpected, since Paré and Houser (1973) reported in their experiment that casualty rats revealed extensive glandular ulcers and that rumenal ulcers were not frequently observed. Other studies from the same laboratory (Paré, 1974, 1975, 1976, 1977a, 1977b; Paré, Natelson, Vincent, \& Isom, 1980) also emphasized the development of glandular, rather than nonglandular, ulcers. Even though all DI and some LE animals developed glandular ulcers in the present study, the noted pathology was not as severe as that found in the studies previously cited. A number of explanations could be presented to account for this difference. These include: (1) age of the animals, (2) survival of all animals and length of testing, (3) habituation period, and (4) lower running activity of all animals.

With reference to age as a factor, the subjects utilized in the present study were older -18 weeks at the beginning of the experiment-than the animals typically employed in previous activity-stress-ulcer experiments. For example, in his 1975 study, Paré used 8-week old animals. Age appears to be a vital factor in determining whether glandular or nonglandular ulcers will develop during starvation. Pfeiffer, Debro, and Muller (1966) demonstrated that starvation primarily induced glandular rather than forestomach ulceration in the weanling rat, but primarily forestomach rather than glandular ulceration in older rats. If, indeed, the animals were selfstarving in the present experiment, the obtained results are quite reasonable.

Concerning survival of animals and length of testing, the fact that all of the animals tested in the present experiment survived the 9 days of testing could also account for noted differences in this study. In other studies cited, most of the animals did not survive, and the few that did survive did not exhibit stomach pathology. Many of the other studies were carried out for a 21-day period. Welker, Garber, and Brooks (1977) demonstrated that when rats were given $1 \mathrm{~h}$ of feeding in successive 48-h intervals, the occurrence of rumenal ulcers peaked at about 7 days and dissipated within 21 days. Animals in the present study were sacrificed on Day 9, which is close to that peak period. In addition, Peters and Boyd (1966) noted that ulcers were observed in the pyloric (glandular) stomach when body-weight loss exceeded $30 \%$. In the present study, body-weight loss was $\mathbf{2 3 . 6 \%}$ for DI animals and $\mathbf{1 8 . 2 \%}$ for LE animals. Glandular ulcers were just beginning to develop at the conclusion of the experiment.

The third factor that could account for the variations noted is the duration and type of habituation that animals experienced. In the present study, the habituation period was 7 days and the animals had constant access to the running wheel. This adaptation to the experimental apparatus may have attenuated glandular ulcer development in the animals. In other studies previously cited, the habituation period was no longer than 5 days, and in some instances there was no habituation period at all. In some experiments, access to the running wheel was blocked, whereas in other cases animals were allowed constant access to the running wheel during habituation.

A fourth factor is running behavior, which, in both DI and LE animals, was considerably lower during the testing session in the present experiment than in previous studies. In the present study, the mean number of revolutions never exceeded 800 in a $24-h$ period. Paré (1975) has reported over 12,000 wheel revolutions in a 24-h period in high-activity rats and over 6,000 wheel revolutions in a 24 -h period in lowactivity rats. This lower running behavior may have attenuated glandular ulcer development in the present study.

In conclusion, the present study demonstrates that DI animals develop more gastric pathology than do control LE animals in the activity-wheel paradigm. The results are not due to differences in running behavior, since DI animals did not run significantly more than LE animals. However, decreases in body weight and water intake may augment the development of gastric pathology in DI animals. It would appear that the lack of vasopressin, or physiological results of this deficit, renders DI animals more sensitive to self-starvation and activity stress than it does control animals.

\section{REFERENCES}

Murphy, H. M., \& Wideman, C. H. Basic alterations in serum levels of several chemical substances in Brattleboro rats. Annals of the New York Academy of Sciences, 1982, 394, 270-274.

PARÉ, W. P. Feeding environment and the activity-stress ulcer. Bulletin of the Psychonomic Society, 1974, 4, 546-548.

Paré, W. P. The influence of food consumption and running activity on the activity-stress ulcer in the rat. American Journal of Digestive Diseases, 1975, 20, 262-273.

Paré, W. P. Activity-stress ulcer in the rat: Frequency and chronicity. Physiology \& Behavior, 1976, 16, 699-704.

PARÉ, W. P. Body temperature and the activity-stress ulcer in the rat. Physiology \& Behavior, 1977, 18, 219-223. (a)

PARÉ, W. P. Gastric secretion and activity-stress lesions in the rat. Journal of Comparative and Physiological Psychology, 1977, 91, 778-783. (b)

Paré, W. P., \& Houser, V. P. Activity and food-restriction effects on gastric glandular lesions in the rat: The activity-stress ulcer. Bulletin of the Psychonomic Society, 1973, 2, 213-214.

Paré, W. P., Natelson, B. H., Vincent, G. P., \& Isom, K. E. A clinical evaluation of rats dying in the activity-stress ulcer paradigm. Physiology \& Behavior, 1980, 25, 417-420.

PARÉ, W. P., \& Temple, L. J. Food deprivation, shock stress and stomach lesions in the rat. Physiology \& Behavior, 1973, 11, 371-375. 
Peters, J. M., \& Boyd, E. M. Organ weights and water levels of the rat following reduced food intake. Journal of Nutrition, 1966, 90, 354-360.

Pfeiffer, C. J., Debro, J. R., \& Muller, P. J. Gastric pathologic and biochemical changes induced by starvation of weanling rats. Life Sciences, 1966, 5, 509-519.

Robert, A., \& Nezamis, J. E. Effect of cortisol on gastric ulcers of the Shay rat. Proceedings of the Society for Experimental Biology and Medicine, 1958, 98, 9-12. (a).

Robert, A., \& Nezamis, J. E. Ulcerogenic property of steroids. Proceedings of the Society for Experimental Biology and Medicine, 1958, 99, 443-447. (b)

Routtenberg, A., \& Kuznesof, A. W. Self-starvation of rats living in activity wheels on a restricted feeding schedule. Journal of Comparative and Physiological Psychology, 1967, 64, 414-421.
Valtin, H. The discovery of the Brattleboro rat, recommended nomenclature, and the question of proper controls. Annals of the New York Academy of Sciences, 1982, 394, 1-9.

Valtin, H., \& Schroeder, H. A. Familial hypothalamic diabetes insipidus in rats (Brattleboro strain). American Journal of Physiology, 1964, 206, 425-430.

Welker, R. L., Garber, J., \& Brooks, F. Stress as a function of irregular feeding of food deprived rats. Physiology \& Behavior, 1977, 18, 639-645.

Wideman, C. H., \& MURPhy, H. M. The effects of restraint and restraint plus intermittent shock on ulcer formation in Brattleboro rats. Physiological Psychology, 1983, 11, 78-80.

(Manuscript received March 28, 1983; revision accepted for publication August 10, 1983.) 